\title{
Large pore isoreticular MOFs as tunable nanoreactors
}

\author{
C.M.L. Vande Velde ${ }^{1}$, R. Fucci ${ }^{1}$, P. Cool $^{2}$ \\ ${ }^{1}$ University of Antwerp, iPRACS, Groenenborgerlaan 171, 2020 Antwerp, Belgium \\ ${ }^{2}$ University of Antwerp, LADCA Universiteitsplein 1, 2610 Wilrijk, Belgium
}

christophe.vandevelde@uantwerpen.be

The development of innovative environmentally friendly catalysts is of crucial importance for the establishment of a new sustainable chemical industry. The immobilization of the catalysts on a support can solve problems of selectivity and activity. We propose a scaffold based on Metal Organic Frameworks (MOFs). Under appropriate conditions they can be assembled into a porous material on which we can immobilize catalyst, making possible its recovery/reuse at the end of the process. The advantages of these scaffolds are clear: uniform, reproducible and controllable manufacture, and the possibility to engineer the linkers. Therefore, we can control and personalize the whole network structure. By using these networks as scaffolds for the immobilization of the catalysts, MOFs turn into actual nanoreactors. Our proposed nanoreactors will be designed and synthesized according to modular principles (based on isoreticular synthesis ${ }^{[1]}$ ). The desired MOFs will have to have some specific characteristics to be used as scaffold for catalyst: pore size in the range of mesopores $(\geq 6 \mathrm{~nm}), 1-\mathrm{D}$ hexagonal structure (channel-like) to help the diffusion of reactants/products ${ }^{[2]}$ and easy/fast/cheap to synthesize in its organic components. In order to achieve the aim of the project, an easy and fast strategy has been optimized for the synthesis of novel tritopic organic linkers (Figure 1).

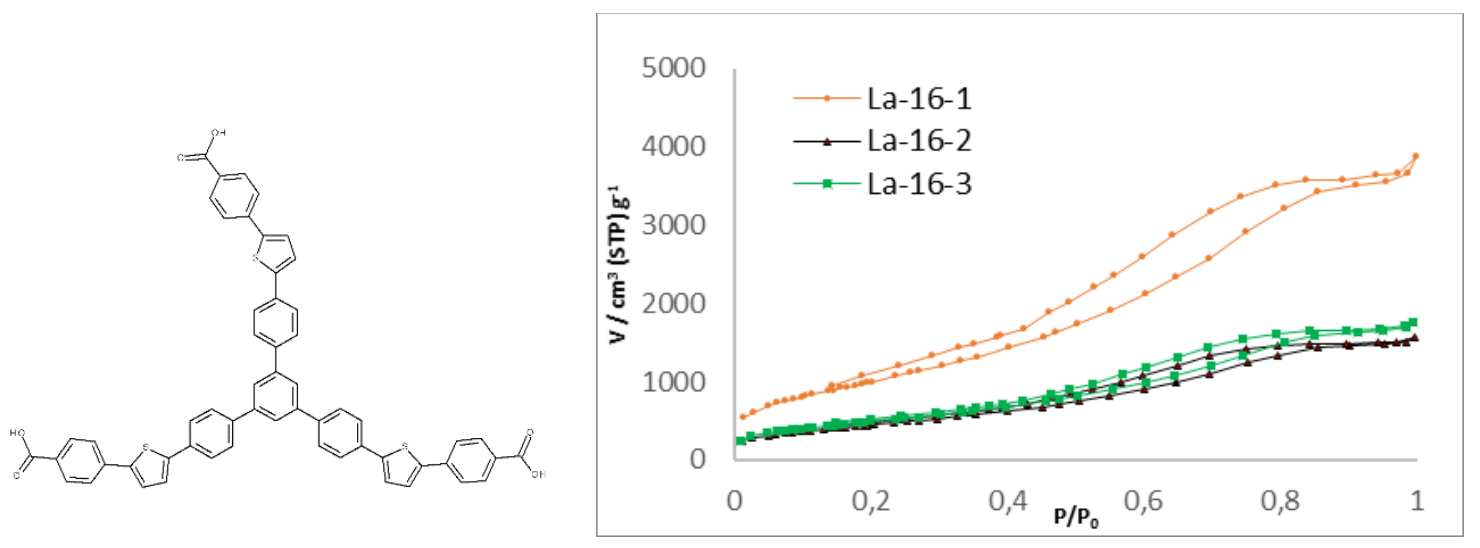

Figure 1. Example linker for mesoporous hexagonal MOFs (left); sorption isotherms for La-MOFs (right)

Conventionally, the synthesis of organic linkers is based on the use of the well-known Suzuki coupling. This approach would require extra synthetic steps. Direct arylation ${ }^{[3]}$, is the coupling of aryl halides with catalytically activated aryl C-H bonds. Therefore, the number of synthetic steps is reduced. The library of long star-shaped linkers is currently used for the synthesis of $\mathrm{Zr}$-, La-, In- and Gabased MOFs, and the first results will be presented here ${ }^{[4]}$.

[1] Deng, H.; et al. Science 2012, 336 (6084), 1018-1023

[2] Čejka, Morris, Nachtigall; RSC Catalysis Series No. 28 (2017)

[3] Yabo Li et al. J. Org. Chem. 2014, 79, 2890-2897

[4] Fucci, Vande Velde; Faraday Discussions, 2021, accepted.

Keywords: catalysis; MOF; linker; isoreticular; large pore

Rosa Fucci, as holder of a PhD grant strategic basic research of the Research Foundation - Flanders with FWO file number IS92420N, thanks the FWO Flanders for funding. 